\title{
Regenerative Medicine
}

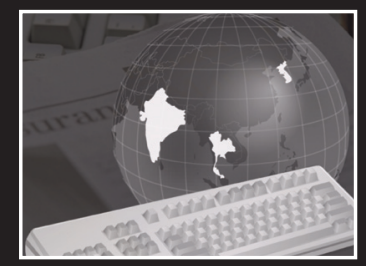

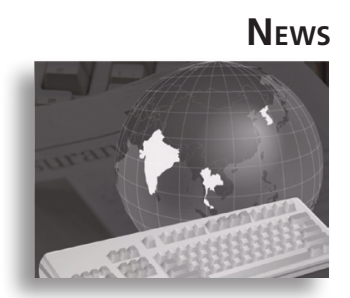

INDUSTRY UPDATE

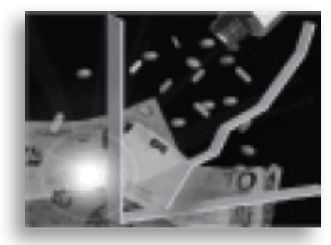

Research Highights

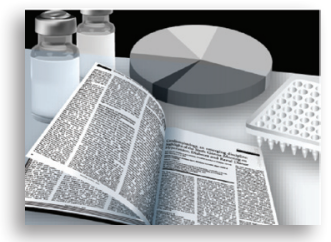

Research suggests that the heart's natural repair mechanisms may be boosted by stem cells

A recent study, published in $E M B O$ Molecular Medicine, has found a potential reason why injecting cardiosphere-derived cells (CDCs) into the heart can regenerate infarcted myocardium. The research team from the Cedars-Sinai Heart Institute (CA, USA) found that the production of existing adult cardiomyocytes and recruitment of existing stem cells, which will mature into cardiomyocytes, is improved by this technique. It is hoped that this finding may help in the development of novel therapeutic strategies that can improve the efficacy of stem cell-based treatments in conditions such as myocardial infarction.

It is well established that the adult mammalian heart is capable of limited endogenous regeneration, and clinical trials have demonstrated that stem cell therapy with CDCs is effective in regenerating the infarcted human heart. However, the reason for this has remained elusive. Therefore, the researchers used a genetic fate-mapping approach and assays to test the cellular origin of this in normal, infarcted and CDC-treated adult mammalian hearts.

The research team found that although the heart's endogenous stem cells contribute to rebuilding heart tissue after myocardial infarction, they are not responsible for the normal replenishment of lost heart cells: in the normal heart, they found that existing cardiomyocytes contribute to the formation of new heart cells (i.e., a new cardiomyocyte will replace a dying one), whereas in an infarcted heart, existing cardiomyocytes also assist in the development of new cells. In stem cell therapy, this effect can be amplified because both the heart's natural response to injury and normal means of cell replacement are improved, as is the heart's function and structure.

This research builds on findings from a recent clinical trial by the same research team, in which patients who underwent an experimental stem cell procedure had reduced myocardial infarction scars when compared with patients who had not undergone the procedure.

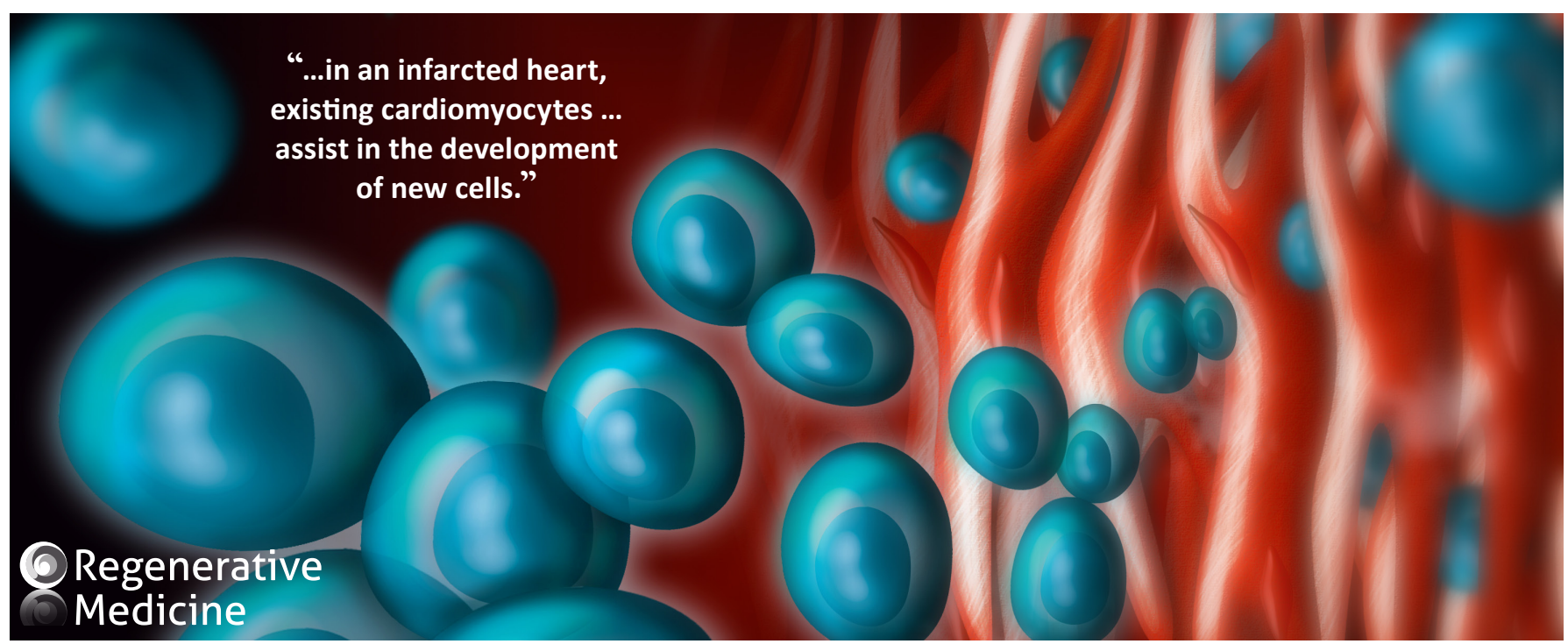


Eduardo Marbán (Cedars-Sinai Heart Institute), senior author, said, "We're finding that the effect of stem cell therapy is indirect. It stimulates proliferation of dormant surviving host heart tissue, and it attracts stem cells already in the heart. The resultant new heart muscle is functional and durable, but the transplanted stem cells themselves do not last long. Understanding the cellular sources and mechanisms of heart regeneration is the first step toward refining our strategies to more effectively regenerate healthy tissue after heart attacks."

By better understanding the process of regenerative cardiomyogenesis, the researchers hope that the efficacy of stem cell-based treatments will be improved and, consequently, repopulation of infarcted myocardium will increase.

- Written by Natasha Leeson

Sources: Malliaras K, Zhang Y, Seinfeld J et al. Cardiomyocyte proliferation and progenitor cell recruitment underlie therapeutic regeneration after myocardial infarction in the adult mouse heart. EMBO Mol. Med. 5(2), 191-209 (2013); Cedars-Sinai Heart Institute press release: http://cedars-sinai.edu/About-Us/News/NewsReleases-2013/Cedars-Sinai-Heart-Institutestudy-stem-cells-boost-hearts-natural-repairmechanisms.aspx

\section{Stem cells show promise for the future of stroke treatment}

A new study from Autónoma University of Madrid (Spain) and Institute of Biomedical Research Alberto Sols (Spain) has claimed that allogenic bone marrow-derived mesenchymal stem cells (BM-MSCs) and adipose-derived mesenchymal stem cells (AD-MSCs) improved brain and nerve repair in rats. It is hoped that the research will contribute to the growing evidence for the use of stem cells in human trials for neurological disorders, such as patients who have suffered strokes.

Strokes occur when blood supply to the brain is compromised, either due to ischemia or hemorrhage. This results in a rapid loss of brain function that can leave some patients unable to walk, talk or see. Stem cells provide a potential treatment option for stroke patients; however, this is yet to be confirmed in clinical trails.

In this study, published recently in Stem Cell Research and Therapy, the researchers used adult rats with permanent middle cerebral artery occlusion as a model to compare the therapeutic potentials of AD-MSCs and BM-MSCs. The rats were randomly assigned to one of four groups (the sham-operated group, the infarct group, the BM-MSC group and the AD-MSC group) and were treated intravenously with either saline or their respective stem cell infusion. The stem cell-treated rats displayed better functional recovery
$24 \mathrm{~h}$ after the stroke, and at the 14-day follow-up, they demonstrated near to normal scores in functional evaluation tests. Furthermore, the stem cell-treated rats also showed decreased cell death and increased cell proliferation. It was observed that the rats displayed these improvements even though there was no migration of the stem cells to the damaged area of the brain. The researchers highlighted that there were no differences in the effects of the treatment between the cells from either source.

One of the authors, Exuperio DíezTejedor, La Paz University Hospital (Spain), was enthusiastic about the outcome of the research: "Improved recovery was seen regardless of origin of the stem cells, which may increase the usefulness of this treatment in human trials. Adipose-derived cells, in particular, are abundant and easy to collect without invasive surgery."

- Written by Natasha Leeson

Sources: Gutierrez-Fernandez M, RodriguezFrutos B, Ramos-Cejudo J et al. Effects of intravenous administration of allogenic bone marrow- and adipose tissue-derived mesenchymal stem cells on functional recovery and brain repair markers in experimental ischemic stroke. Stem Cell Res. Ther. 4(1), 11 (2013); Medical News Today press release: www.medicalnewstoday.com/releases/255492.php 


\section{Blind mice have retinas}

\section{regenerated}

Researchers at the University of Oxford (UK) have reported that they have been able to reform the entire light-sensitive layer of the retina in mice that were completely blind. This research shows the potential of restoring vision using cell transplantation and may one day be applicable in treating patients with degenerative eye disease, such as retinitis pigmentosa.

Retinitis pigmentosa is an inherited, degenerative eye disease that can often lead to blindness. It is thought that this is due to the outer nuclear layer progressively losing photoreceptor cells. Previous studies have demonstrated that vision can be restored, even after photoreceptors have been lost, in previously blind patients after stimulation by subretinal electrodes. However, photoreceptor replacement in the absence of the host outer nuclear layer has not been investigated as thoroughly. The researchers in this study wanted to determine whether a functional outer nuclear layer could be reconstituted de novo by precursor cell transplantation, instead of adding more photoreceptor cells into a pre-existing host outer nuclear layer.

The study, published in Proceedings of the National Academy of Sciences USA, details how the scientists injected rod precursor cells into completely blind mice, which acted as the model for severe human retinitis pigmentosa. The rod precursors were able to reform into an appropriately polarized outer nuclear layer with light-sensitive outer segments and form connections with host neurons downstream. By comparing visual function in the mice before and after transplantation, the scientists observed that visual function was restored in mice with absolutely no rod function at baseline.

One of the authors of the study, Robert MacLaren, from the University of Oxford, explained: "We have recreated the whole structure, basically it's the first proof that you can take a completely blind mouse, put the cells in and reconstruct the entire light-sensitive layer."

The authors surmise that a new and functional photoreceptor layer can be created by transplanting rod precursors into degenerate outer retinas. They hope that their data may be used in supporting the potential clinical application of photoreceptor precursors in the treatment of human blindness due to conditions such as retinitis pigmentosa.

- Written by Natasha Leeson

Sources: Singh MS, Charbel Issa P, Butler R et al. Reversal of end-stage retinal degeneration and restoration of visual function by photoreceptor transplantation. Proc. Natl Acad. Sci. USA 110(3), 1101-1106 (2013); BBC news: 'Totally blind mice get sight back': www.bbc.co.uk/news/ health-20898935

\section{Induced pluripotent stem cells could be used in cell therapy without eliciting immune rejection}

A team of researchers from the Boston University School of Medicine (MA, USA) have recently published a study in the journal Cell Stem Cell demonstrating that they were able to transplant tissues derived from induced pluripotent stem cells (iPSCs) into genetically identical recipients, without eliciting immune rejection. This could potentially be very useful in the treatment of numerous conditions.

It has been previously found that even though iPSCs can be made in a patient-specific manner, undifferentiated mouse iPSCs can be immunogenic upon
"This research shows the potential of restoring vision using cell transplantation and may one day be applicable in treating patients with degenerative eye disease..."


"...future work should be directed at assessing whether tissues derived from human induced pluripotent stem cells will similarly lack immunogenicity." transplantation. This has affected the applicability of using autologous iPSCs in cell-replacement therapy. Therefore, the research team wanted to assess the immunogenicity of therapeutically relevant cell types that could one day be used in transplantation.

In this study, the scientists derived mice iPSCs from adult cells in an experimental model. The iPSCs were differentiated into three cell types (hepatocytes [liver], neuronal [nerve] and endothelial [blood vessel lining] cells) to reflect the body's tissue and organ systems. By transplanting the iPSCs into a genetically identical experimental model, the researchers were able to test whether the cells would illicit an immune response, one that may be similar to those seen in a clinical application. They found that there was no increased T-cell proliferation in vitro, nor an antigen-specific secondary immune response. Furthermore, they found little evidence to support the claim of an immune response to undifferentiated, syngeneic iPSCs.

Currently, immunosuppressive drugs are prescribed clinically to counteract immune rejection in transplantation; however, their usage can increase the patient's risk of developing cancer. One of the study's senior authors, Ashleigh Boyd, who is an assistant professor of dermatology at the Boston University School of Medicine, explained: "If the use of immunosuppressive drugs can be avoided, as may be the case for patient-specific iPSC-based therapies, it would be preferable. Our results are very promising and future work should be directed at assessing whether tissues derived from human iPSCs will similarly lack immunogenicity."

- Written by Natasha Leeson

Sources: Guha P, Morgan JW, Mostoslavsky $G$, Rodrigues NP, Boyd AS. Lack of immune response to differentiated cells derived from syngeneic induced pluripotent stem cells. Cell Stem Cell doi:10.1016/j.stem.2013.01.006. (2013) (Epub ahead of print); Boston University School of Medicine press release: www. bumc.bu.edu/2013/01/28/busm-study-showspotential-of-differentiated-ips-cells-in-celltherapy-without-immune-rejection

\title{
Tissue engineers report knee cartilage repair success with new biomaterial
}

\author{
Experimental hydrogel scaffold benefits cartilage repair.
}

A recent study, performed at Johns Hopkins University School of Medicine's Translational Tissue Engineering Center (MA, USA), has produced promising data on the benefits of a novel hydrogel scaffold in postsurgical cartilage repair in the knee. The work, described in an article published in Science Translational Medicine, outlines the John Hopkins team's experience with their poly(ethylene glycol) diacrylate hydrogel, which they incorporated into conventional microfracture surgery on the knee. In conventional microfracture surgery to repair damaged cartilage, the bone underneath the damaged tissue has tiny fractures created in it by the surgical team; this leads to the development of fresh cartilage growth, which can repair the existing damaged tissue. However, the regrown cartilage is sometimes weaker than the original material it is intended to replace and sometimes only limited regrowth occurs.

After a period of laboratory experiments and tests performed in goats, the research team pursued an initial clinical trial of the hydrogel in 15 patients. In the patients that received the hydrogel treatment, the gel and an adhesive implant were implanted in addition to the microfracture treatment. A microfracture-only treatment group was also included as a control. At a 6-month postsurgical analysis, MRI demonstrated that the patients that received the hydrogel had significantly higher levels of cartilage regrowth 
compared with the control patients with no major adverse events occurring. In addition, treated patients had less pain than control patients, and knee function (measured using International Knee Documentation Committee scores) increased to comparable levels between the two groups.

Commenting on the results of the study, Jennifer Elisseeff, from Johns Hopkins University School of Medicine's Translational Tissue Engineering Center, said, "Our pilot study indicates that the new implant works as well in patients as it does in the lab, so we hope it will become a routine part of care and improve healing." The trial has enrolled more patients and is continuing while the team are aiming to create a second-generation implant with the hydrogel and adhesive combined into a single implant.

- Written by Sean Fitzpatrick

Sources: Sharma B, Fermanian S, Gibson M et al. Human cartilage repair with a photoreactive adhesive-hydrogel composite. Sci. Transl. Med. 5(167), $167 r a 6$ (2013); Johns Hopkins University School of Medicine: www.hopkinsmedicine. org/news/media/releases/tissue_engineers_ report_knee_cartilage_repair_success_with_ new_biomaterial

\section{About the News}

The News highlights some of the most important events and research. If you have newsworthy information, please contact: Charlotte Barker, Commissioning Editor, Regenerative Medicine,

Future Medicine Ltd, Unitec House, 2 Albert Place, London, N3 1QB, UK

Tel.: +44 (0)20 8371 6090; Fax: +44 (0)20 8343 2313;

c.barker@futuremedicine.com 